This item was submitted to Loughborough's Institutional Repository (https://dspace.lboro.ac.uk/) by the author and is made available under the following Creative Commons Licence conditions.

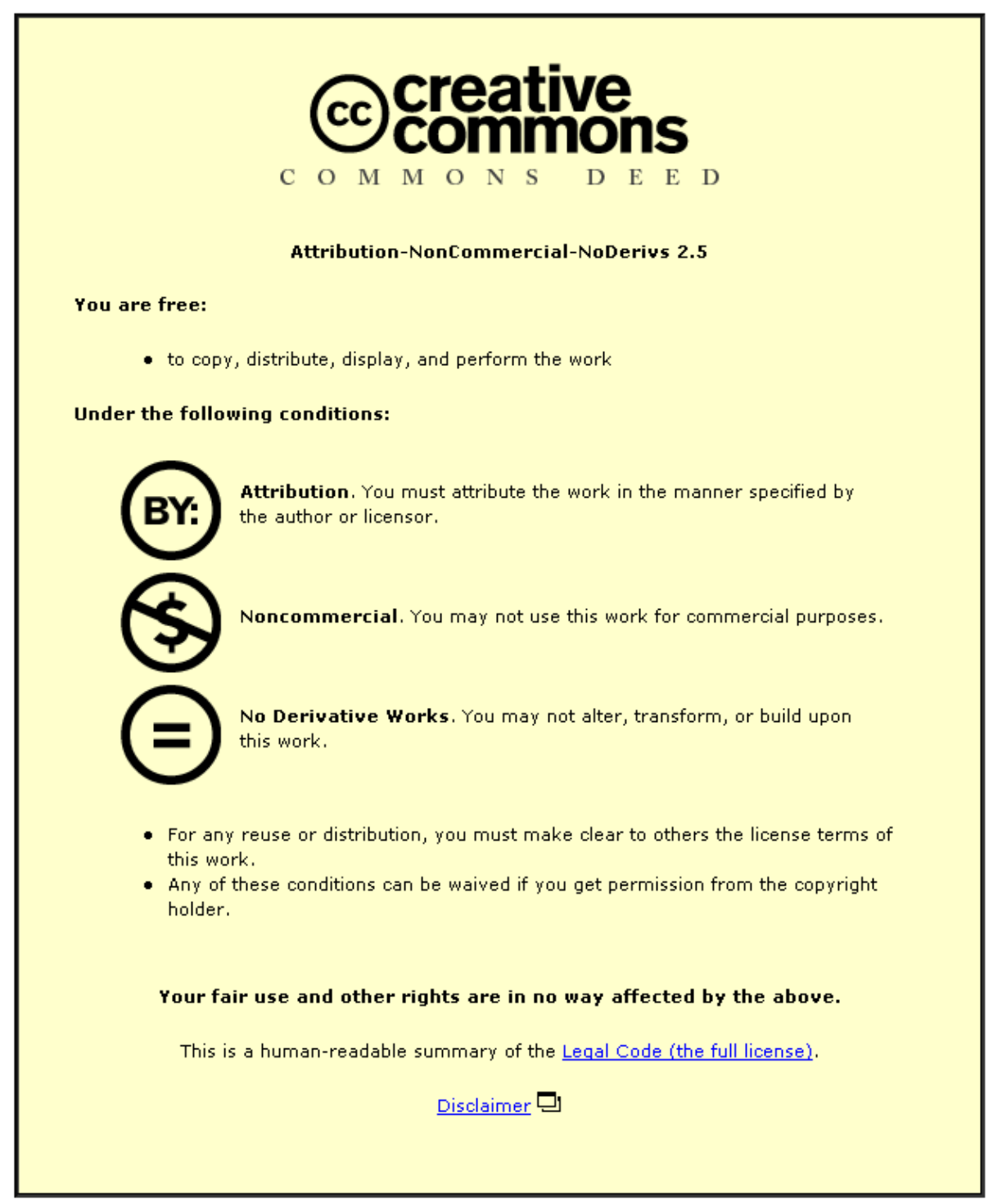

For the full text of this licence, please go to: http://creativecommons.org/licenses/by-nc-nd/2.5/ 


\title{
AGGREGATION EFFECTS IN THE CAKE FILTRATION OF INTERACTING BINARY MIXTURES
}

\author{
K. Chellappah ${ }^{1,2}$, E.S. Tarleton ${ }^{1}$ (e.s.tarleton@lboro.ac.uk) and R.J. Wakeman ${ }^{1,3}$ \\ ${ }^{1}$ Dept. Chemical Engineering, Loughborough University, Loughborough, Leics., LE11 3TU, UK. \\ ${ }^{2}$ BP Exploration, Chertsey Road, Sunbury-on-Thames, TW16 7LN, UK. \\ ${ }^{3}$ Consultant Chemical Engineer, Exeter, EX5 1DD, UK.
}

\begin{abstract}
New data are reported on the filtration of binary fibre/titania (rutile) mixtures. The combined use of the Kozeny-Carman equation and Darcy's law is presented and discussed in relation to application in the filtration of binary mixtures. Upon close study, this approach is shown to have its limitations, particularly when significant aggregation takes place between the two solids species. The difficulties in overcoming these limitations from a purely fundamental basis are highlighted, and a semi-empirical model is presented and discussed. This model appears to represent the specific resistance trend with solids composition reasonably well for a wide range of binary mixture filtration data.
\end{abstract}

\section{KEYWORDS}

Agglomeration; Filtration; Porous media; Packed bed; Mathematical modelling; Colloidal phenomena.

\section{INTRODUCTION}

Previous works on the cake filtration of binary mixtures include those of Shirato et al. (1963), Abe et al. (1979, 1982), Wakeman (1996), Iritani et al. (2002) and Chellappah et al. (2009a, 2009b, 2010). Although considerable work (Koenders and Wakeman, 1997; McDonogh et al., 1992; Wakeman et al., 1991; Wakeman and Akay, 1997) has reported on the effects of physico-chemical interactions on filtration behaviour, as Iritani et al. (2002) point out, there is scarce study on the effects of physico-chemical factors on binary suspension filtrations. There is also sparsity in the study of the filtration of fibre/particle binary mixtures, although the need to dewater such mixtures often arises in industry.

The permeability of a filter cake is the most important factor in cake filtration (in relation to design and scale-up) and is often interpreted through a measure of the cake's specific resistance. Models for cake formation are based on Darcy's law, which uses a single parameter (the permeability) to account for the characteristics of a porous filter cake in so far as they affect fluid flow. There have been many attempts to relate permeability to geometric considerations of a porous medium. One of the most widely used of the resultant theories is the Kozeny-Carman. In this theory, fluid flowing through a porous body loses energy where it is in contact with the internal surfaces of the body; these internal surfaces possess a very complex geometry which cannot be easily described mathematically, but for practical purposes can be interpreted through the body's porosity and specific surface. The Kozeny-Carman equation has important uses for determining the specific surface of particle samples from permeability data and in correlating resistance data for fluid flow through porous media (Wakeman and Tarleton, 2005).

In this paper, physico-chemical interactions with binary fibre/titania (rutile) mixtures are highlighted. The combined use of the Kozeny-Carman equation and Darcy's law is presented and discussed in relation to application in the filtration of binary mixtures. A semi-empirical equation is proposed to represent aggregation phenomena and/or changes in packing behaviour with binary filter cakes. 


\section{THEORETICAL DEVELOPMENT}

Abe et al. (1979) had previously derived a model to predict the average specific cake resistance of binary mixtures based on the Darcy and Kozeny-Carman equations. The model derivation is briefly presented here with further development and relevant elucidation, starting from the KozenyCarman equation:

$\Delta P=\frac{5 S_{m}{ }^{2}\left(1-\varepsilon_{a v}\right)^{2}}{\varepsilon_{a v}{ }^{3}} L u \mu$

and Darcy's law, where the pressure drop across the filter cake can also be expressed as:

$$
\Delta P=u \mu \alpha_{a v} w
$$

Also, the height of the filter cake is given by:

$$
L=\frac{v}{1-\varepsilon_{a v}}
$$

and $v / w$ is the inverse of the binary filter cake true density $\left(\rho_{m}\right)$, expressed by equation (4):

$$
\frac{v}{w}=\frac{1}{X_{D} \rho_{s 1}+\left(1-X_{D}\right) \rho_{s 2}}
$$

Substituting (2), (3), and (4) into (1) and algebraically manipulating gives:

$$
\alpha_{a v}=\frac{5\left(1-\varepsilon_{a v}\right)}{\varepsilon_{a v}{ }^{3}\left(X_{D} \rho_{s 1}+\left(1-X_{D}\right) \rho_{s 2}\right)} S_{m}{ }^{2}
$$

Assuming an 'additive' mechanism, the specific surface of the binary mixture at a given solids composition can be expressed in terms of the effective specific surface of the two solids components:

$$
S_{m}=X_{D} \frac{S_{1}}{\varphi_{1}}+\left(1-X_{D}\right) \frac{S_{2}}{\varphi_{2}}
$$

Equation (6) assumes that with mixtures, the pure component specific surfaces remains unchanged and any changes in total specific surface is attributed only to the changes in volume of the two solids species. Equation (6) does not account for any changes in packing and/or interaction effects between the two solids species in the binary mixture. The terms $S_{1} / \varphi_{1}$ and $S_{2} /$ $\varphi_{2}$ describe the specific surface of the pure fibres and rutile in contact with the fluid permeating through the binary cakes as determined from the pure component filtration data using:

$$
\frac{S_{i}}{\varphi_{i}}=\sqrt{\frac{\alpha_{a v, i} \varepsilon_{a v, i}{ }^{3} \rho_{s i}}{5\left(1-\varepsilon_{a v, i}\right)}}
$$

where $i=1$ for the first (larger) or 2 for the second (larger) solids component. 
No relationship between specific surface and particle size is assumed and $\varphi_{i}$ is termed a shape factor. Also, the term $S_{i} / \varphi_{i}$ is kept as a ratio and not broken up since little is known about the exact relative magnitudes of either $S_{i}$ or $\varphi_{i} . \varphi_{i}$ can be thought of as a lumped parameter taking into account sphericity, surface roughness, as well as pure component size distribution and surface interactions. Hence, $S_{i}$ can be considered to be the specific surface that corresponds to idealised spheres. As pointed out by Donohue and Wensrich (2009), although a shape factor can be used to account for irregular particles, it must be determined empirically. Furthermore, the ratio of the two solids specific surfaces is a reoccurring term and is termed $S_{B}$, expressed as:

$$
S_{B}=\frac{S_{1} / \varphi_{1}}{S_{2} / \varphi_{2}}
$$

It should be noted that $S_{B}$ as defined in equation (8) refers to the ratio of pure component specific surfaces, with a pure component's specific surface determined from equation (7). However, a pure component's effective specific surface may vary in the presence of another solids component due to physico-chemical effects and/or varying packing mechanisms.

\section{MATERIALS, APPARATUS AND EXPERIMENTAL METHOD}

The fibres used were in the form of tissue paper (Merton Cleaning Supplies (Leicester) Ltd). The titania was in the form of the rutile polymorph and obtained from Huntsman. Some relevant properties of the fibres and rutile are summarised in Table 1. $\zeta$-potential values quoted are over the experimental $\mathrm{pH}$ range of interest. The fibres display a much wider size distribution than rutile. $\zeta$-potential measurements suggest $\mathrm{Na}^{+}$to be an indifferent electrolyte and indicate physical adsorption of $\mathrm{Ca}^{2+}$ ions onto the fibre and rutile surfaces. The ionic strength $(I)$ of $0.2 \mathrm{M} \mathrm{NaCl}$ and $0.1 \mathrm{M} \mathrm{CaCl}_{2}$ solutions were 0.2 and 0.6 respectively, as calculated using the Debye-Hückel theory (Atkins, 1998):

$I=\frac{1}{2} \sum_{j} z_{j}^{2} b_{j}$

where $z_{j}$ is the charge number of an ion $j$ and $b_{j}$ its concentration. Well mixed suspensions consisting of either pure fibres, or rutile, or binary mixtures of the two; in deionised (DI) water, 0.2 $\mathrm{M} \mathrm{NaCl}$, or $0.1 \mathrm{M} \mathrm{CaCl}_{2}$ solutions were used in experiments. In both filtration and sedimentation experiments the total solids concentration was maintained at $1.1 \% \mathrm{v} / \mathrm{v}$. Typical SEM images of the rutile and fibres, as well as a more detailed description of solids characterisation and suspension preparation has been previously reported (Chellappah et al., 2009a; 2009b).

An automated pressure filtration apparatus was used for the filtration experiments. Further details on the apparatus have been described elsewhere (Tarleton and Hadley, 2003; Tarleton, 2008). Further details on the filtration and sedimentation experiments, including methodology and some initial results, have been previously reported by Chellappah et al. (2009a)

\section{EXPERIMENTAL RESULTS AND DISCUSSION}

All filtration experiments were performed at constant pressure and analysed to establish values of average specific cake resistance $\left(\alpha_{a v}\right)$ and average cake porosity $\left(\varepsilon_{a v}\right)$, and sedimentation experiments were analysed to establish values of initial settling rate and proportion of sludge, in the manner previously described (Chellappah et al., 2009a). The effects of solids composition on binary suspension filtration are presented in terms of the variable $X_{D}$ which is defined as the ratio 
of the volume of fibres to the total volume of solids in the suspension; $X_{D}=0$ indicates a pure rutile suspension (i.e. no fibres) whereas $X_{D}=1$ indicates a pure fibre suspension (i.e. no rutile).

Figure 1 shows some sedimentation data to help interpret the filtration results. It is seen from Figure 1 that pure rutile and pure fibres in deionised water appear to be stable suspensions which do not readily settle. With only small amounts of fibres added to a pure rutile suspension, rutilefibre interactions take place and the suspension de-stabilises. The general sedimentation trends for suspensions in deionised water and $\mathrm{CaCl}_{2}$ solution appear to be similar. Initial settling rates in the range $0.1<X_{D}<0.8$ were lower for suspensions in $\mathrm{CaCl}_{2}$ solution, presumably due to the formation of low density, loosely networked aggregates. The presence of more loosely networked aggregates in $\mathrm{CaCl}_{2}$ solution was evidenced by the greater proportions of sludge at a given $X_{D}$. Also, it is not surprising that the proportion of sludge data suggests that the fibres form more loosely networked packings than rutile.

Figures 2 and 3 illustrate the effects of solids composition and pressure on $\varepsilon_{a v}$ and $\alpha_{a v}$ for deionised water filtrations. Figures 4 and 5 illustrate the effects of solids composition and solution environment on $\alpha_{a v}$ and $\varepsilon_{a v}$ for $450 \mathrm{kPa}$ filtrations from deionised water as well as from $0.2 \mathrm{M} \mathrm{NaCl}$ and $0.1 \mathrm{M} \mathrm{CaCl}_{2}$ solutions. It is seen from Figures 2-5 that the effects of pressure are less evident relative to the effects of solids composition and solution environment. Some discussions on the effects of solids composition and filtration pressure have been previously published (Chellappah et al., 2009a), where the minima in $\alpha_{a v}$ over the solids composition range $0.3<X_{D}<0.7$ (Figure 3 ) was attributed to rutile-fibre aggregation. Rutile-fibre aggregation was noted visually and is evidenced by sedimentation data. The $\alpha_{a v}$ vs. $X_{D}$ trend for suspensions in deionised water generally corresponds to the trend of initial settling rate vs. $X_{D}$ (Figure 1 ), and this is not surprising as the initial settling rates were largely influenced by the degree of aggregation.

Considering the relationships between the parameters $\alpha_{a v}$ and $\varepsilon_{a v}$ with fibre fraction gives rise to an apparent anomaly, since, as suggested by the Kozeny-Carman and Happel cell models (Wakeman and Tarleton, 2005), an increase in porosity generally results in an increased permeability (lower $\left.\alpha_{a v}\right)$. However, it should be recognised that these models only provide reasonable approximations if the solids form remains similar. The fibres and rutile are of very different forms, with the rutile being more isometric in shape, and this may result in varying packing mechanisms moving from pure rutile to pure fibre cakes. Also, the rutile-fibre interactions may further influence the specific surface exposed to fluid flow at the various solids compositions, which in turn will affect the specific resistance.

In terms of packing, the additive porosity model (Shirato et al., 1963) gave reasonable representations of porosity data for filtrations from deionised water:

$$
\varepsilon_{a v}=\frac{\frac{\varepsilon_{a v, 1} X_{D}}{1-\varepsilon_{a v, 1}}+\frac{\varepsilon_{a v, 2}\left(1-X_{D}\right)}{1-\varepsilon_{a v, 2}}}{\frac{X_{D}}{1-\varepsilon_{a v, 1}}+\frac{1-X_{D}}{1-\varepsilon_{a v, 2}}}
$$

As an illustrative example, the $\varepsilon_{a v}$ vs. $X_{D}$ trend given by equation (10) for $450 \mathrm{kPa}$ filtrations from deionised water is included in Figure 5 . The additive law represented the experimental porosity data better than typical interparticle penetration models (which generally show a minimum porosity at some intermediate mixture fraction), perhaps indicating that rutile particles coat fibre surfaces (reducing the interfibre porosity to a small extent) instead of more intensively filling up interfibre voids (Chellappah et al., 2009a).

In general, the $0.1 \mathrm{M} \mathrm{CaCl}_{2}$ solution environment had a marginally greater effect on the filtration behaviour relative to the $0.2 \mathrm{M} \mathrm{NaCl}$ solution environment and this could be due to: (1) $\mathrm{Ca}^{2+}$ ions 
physically adsorbing onto the solids surfaces whereas $\mathrm{Na}^{+}$ions behaved as an indifferent electrolyte, and/or (2) the $0.1 \mathrm{M} \mathrm{CaCl}_{2}$ solution being of a greater ionic strength. Taking into account that the fibres and rutile were oppositely charged in $0.1 \mathrm{M} \mathrm{CaCl}_{2}$ solution, the effect of $\mathrm{CaCl}_{2}$ solution environment on rutile-fibre interactions could be expected to be greater than on the pure component interactions. However, there was little variation in $\varepsilon_{a v}$ (Figure 5) and $\alpha_{a v}$ (Figure 4) due to a different solution environment in the solids composition range $0.3<X_{D}<0.7$. This apparent discrepancy may be explained by the fact increasing states of aggregation cannot be expected to continually decrease $\alpha_{a v}$ and increase $\varepsilon_{a v}$ as there will eventually be a limit under a given applied pressure. This limit seems to have already been reached by cakes formed from suspensions in deionised water over the solids composition range $0.3<X_{D}<0.7$. It is hence postulated that, up to a certain extent, interaction (aggregation) between the two solids components will strongly affect a binary cake's average specific resistance via decrease in specific surface and/or increase in porosity, with the magnitude of the effects varying with solids composition and solution environment.

\section{MODELLING ANALYSIS}

Before direct application of the model (derived above) to experimental data, some general characteristics are discussed, in particular the potential for representing a minimum in specific resistance at some intermediate solids composition and the significance of aggregation effects.

\section{Study of Model Characteristics}

From equations (5) and (6), and assuming the hypothetical case of constant $\varepsilon_{a v}, S_{1} / \varphi_{1}$ and $S_{2} / \varphi_{2}$ (invariant with solids composition), equating the differential of $\alpha_{a v}$ to 0 (a minimum can only occur when the differential is equal to 0 ) leads to an interesting outcome. After relevant manipulation and the use of equation (8), it is seen that a minimum in $\alpha_{a v}$ at some intermediate solids composition (assuming constant porosity and specific surface) would only occur if:

$$
\frac{2 \rho_{s 1}}{\rho_{s 1}+\rho_{s 2}}<S_{B}<\frac{\rho_{s 1}+\rho_{s 2}}{2 \rho_{s 2}}
$$

Combining equations (5) and (6) and taking into account variations in $\varepsilon_{a v}$ using equation (10), but assuming constant $S_{1} / \varphi_{1}$ and $S_{2} / \varphi_{2}$ (specific surface invariant with solids composition) gives:

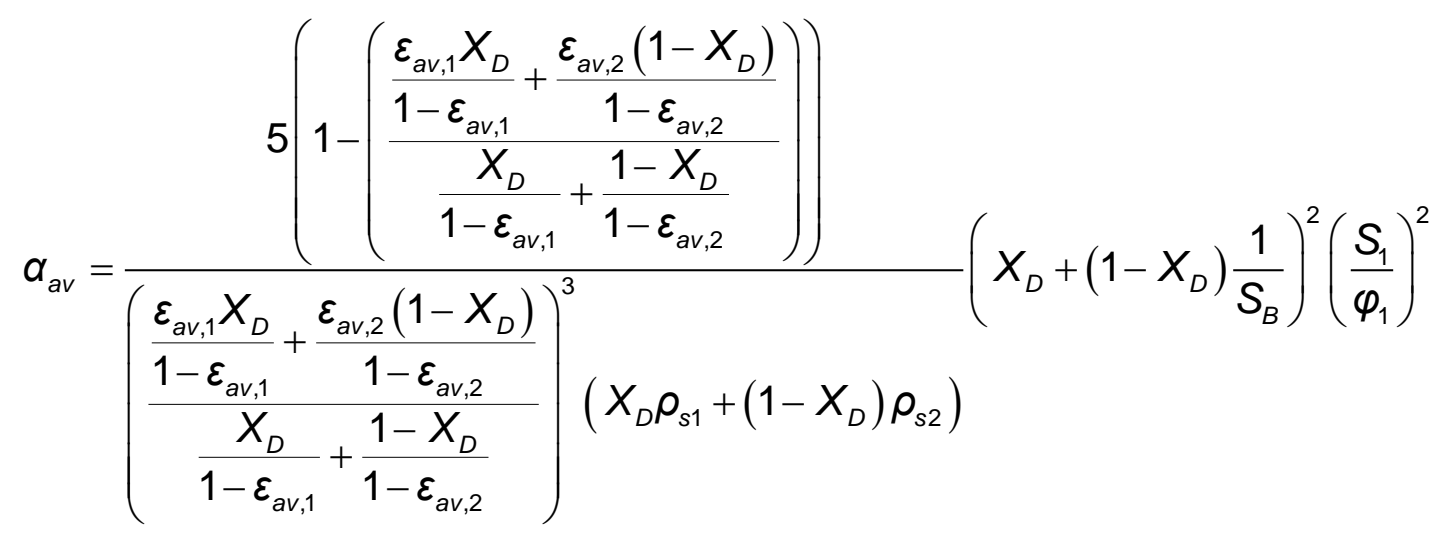

From equation (12) (assuming constant $S_{1} / \varphi_{1}$ and $S_{2} / \varphi_{2}$ ), equating the differential of $\alpha_{a v}$ to 0 and carrying out relevant manipulation shows that a minimum in $\alpha_{a v}$ would only occur if: 


$$
\frac{1}{\left(1.5 \frac{\varepsilon_{a v, 2}}{\varepsilon_{a v, 1}}-1\right)\left(\frac{1-\varepsilon_{a v, 1}}{1-\varepsilon_{a v, 2}}\right)+0.5 \frac{\rho_{s 2}}{\rho_{s 1}}}<S_{B}<\left(1.5 \frac{\varepsilon_{a v, 1}}{\varepsilon_{a v, 2}}-1\right)\left(\frac{1-\varepsilon_{a v, 2}}{1-\varepsilon_{a v, 1}}\right)+0.5 \frac{\rho_{s 1}}{\rho_{s 2}}
$$

Equation (13) is another interesting result as it outlines the range of $S_{B}$, in terms of the two pure solids densities and pure component $\varepsilon_{a v}$, within which a minimum or maximum in $\alpha_{a v}$ could occur at some intermediate solids composition. This equation should, in theory, hold for any binary mixture where the effective specific surface values of the two solids components do not vary with solids composition and the $\varepsilon_{a v}$ vs. solids composition trend can be satisfactorily represented by the additive porosity model.

Using data from $450 \mathrm{kPa}$ filtrations of fibre/rutile mixtures in deionised water as an example, equation (11) will simplify to (a minimum in $\alpha_{a v}$ will only occur if) $0.47<S_{B}<0.66$. Using the same dataset, equation (13) will simplify to $0.58<S_{B}<1.73$, indicating that accounting for the porosity variation widens the limits within which a minimum in $\alpha_{a v}$ at some intermediate solids composition could be obtained. It is noted that data at other trialled pressures show similar limits. According to equations (7) and (8), the limits of the expressions represented in equations (11) and (13) are not met experimentally if $S_{B}$ values are calculated from the pure component filtration data. From the values calculated using data from pure component filtrations with deionised water, $S_{B}$ was approximately $3.7,4.9$ and 5.5 for filtrations at 150,450 and $600 \mathrm{kPa}$, respectively.

\section{Significance of Aggregation}

As an illustrative example, a family of curves are plotted with first $S_{1} / \varphi_{1}$ and then $S_{2} / \varphi_{2}$ altered such that $S_{B}$ ranges from 0.65 to 1.45 (Figure 6). For the purpose of this illustrative example, curves (a), (b) and (c) were plotted using the value of $S_{2} / \varphi_{2}$ as determined from equation (7) with experimental data from a $450 \mathrm{kPa}$ filtration of a pure rutile suspension in deionised water. Values of $S_{1} / \varphi_{1}$ were varied such that $S_{B}$ ranged from 0.65 to 1.45 . Similarly, for curves (d), (e) and (f), $S_{1} / \varphi_{1}$ was determined from equation (7) with experimental data from a $450 \mathrm{kPa}$ filtration of a pure fibre suspension in deionised water. Values of $S_{2} / \varphi_{2}$ were varied such that $S_{B}$ ranged from 0.65 to 1.45 . The pure component porosities used were from the $450 \mathrm{kPa}$ pure component filtrations with deionised water. Since the values of $S_{B}$ were within the range outlined by equation (13) for the corresponding data, minima in specific resistance were obtained at some intermediate solids composition. However, the minima shown are not as pronounced as that obtained experimentally (compare Figures 3 and 6). This apparent discrepancy may not be surprising since, as noticed visually and evidenced by the sedimentation data (where both pure component suspensions do not settle but settling rates are significantly increased for mixtures), the physical nature of the binary suspensions are altered synergistically. An analysis of the behaviour of an interacting binary suspension cannot be made by a simplistic extrapolation from the known behaviour of the two pure component suspensions.

It should be noted that if the effective specific surface of rutile and/or fibres vary with solids composition due to aggregation and/or changes in packing behaviour, so $S_{B}$ may also vary with solids composition. In the analysis so far it has been assumed that $S_{B}$ remains constant and can be determined from the pure component filtrations. Further, the significance of $S_{B}$ in the analysis of binary mixture filtration is highlighted via equations (11) and (13) and Figure (6). For $S_{B}$ values to be the basis of a meaningful study, it will have to represent the true ratio of effective specific surfaces at any given solids composition. It is difficult to determine a suitable method of fundamentally accounting for the variation of specific surface of the two solids species with solids composition, due to the inherent complexities potentially brought about by aggregation and varying packing mechanisms at the different solids compositions. To overcome these limitations, a fitting procedure has been carried out on the total solids effective specific surface. 


\section{Model Fitting}

Representations of $S_{m}$ at the different solids compositions were experimentally determined by rearranging equation (5) to give:

$$
S_{m}=\sqrt{\frac{\alpha_{a v} \varepsilon_{a v}{ }^{3}\left(X_{D} \rho_{s 1}+\left(1-X_{D}\right) \rho_{s 2}\right)}{5\left(1-\varepsilon_{a v}\right)}}
$$

Equation (6) gives an 'additive' type approach to describing the variation of total effective specific surface with solids composition, and only accounts for the variation in total specific surface brought about by progressively 'removing' a given volume of solids component 1 and 'replacing' it with solids component 2 of a similar volume. Where the two solids are well mixed and of significantly different size distributions and/or shapes (more likely to result in packing variations), and in particular where interactions between the two solids occur, then equation (6) may not be appropriate. Instead, equation (15) was used to represent the variation of total solids effective specific surface:

$$
S_{m}=X_{D} \frac{S_{1}}{\varphi_{1}} \exp \left(-b_{1}\left(1-X_{D}\right)\right)+\left(1-X_{D}\right) \frac{S_{2}}{\varphi_{2}} \exp \left(-b_{2} X_{D}\right)
$$

where $b_{1}$ and $b_{2}$ are fitting parameters. A curve fitting process was done to determine $b_{1}$ and $b_{2}$, using non-linear regression on the values of $S_{m}$ as determined from equation (14). $S_{1} / \varphi_{1}$ and $S_{2} / \varphi_{2}$ (pure component specific surfaces) were determined using the pure component filtration data and equation (7).

The exponential functions were used to describe variations in total specific surface due to aggregation and variations in packing behaviour, in the absence of a more fundamental model, with the fitting parameters $b_{1}$ and $b_{2}$ giving a quantitative estimate of the effects especially at both extremes of solids composition by behaving as 'lumped coefficients'. The fitting parameter $b_{1}$ is a coefficient for describing the effects of the addition of small solids on the larger solids component's effective specific surface (due to changes in packing and interactions). Correspondingly, the fitting parameter $b_{2}$ is a coefficient for describing the effects of the addition of larger solids on the small solids component's effective specific surface. For example, as the cake becomes richer in the small solids component ( $X_{D}$ decreases), then the term 'exp $\left(-b_{2} X_{D}\right)$ ' in equation (15) becomes less significant indicating that the influence the larger solids component has on the small solids component's effective specific surface becomes less significant. However, the term ' $\left(1-X_{D}\right)\left(S_{2} / \varphi_{2}\right)$ ' becomes greater due to the increasing volume of the small solids component in the cake. Further, the greater the magnitude of $b_{2}$, the more of an effect the term ' $\exp \left(-b_{2} X_{D}\right)$ ' will have on the term ' $\left(1-X_{D}\right)\left(S_{2} / \varphi_{2}\right) \exp \left(-b_{2} X_{D}\right)$ '.

Substituting equation (15) into equation (5) gives:

$$
\alpha_{a v}=\frac{5\left(1-\varepsilon_{a v}\right)}{\varepsilon_{a v}{ }^{3}\left(X_{D} \rho_{s 1}+\left(1-X_{D}\right) \rho_{s 2}\right)}\left(X_{D} \frac{S_{1}}{\varphi_{1}} \exp \left(-b_{1}\left(1-X_{D}\right)\right)+\left(1-X_{D}\right) \frac{S_{2}}{\varphi_{2}} \exp \left(-b_{2} X_{D}\right)\right)^{2}
$$

Figure 7 shows the fits given by equation (16) to the filtration data for fibre/rutile mixtures in deionised water at 150, 450 and $600 \mathrm{kPa}$, where curves representative of the data are presented. The model was also fitted to data for $450 \mathrm{kPa}$ filtrations of fibre/rutile mixtures in $0.2 \mathrm{M} \mathrm{NaCl}$ and $0.1 \mathrm{M} \mathrm{CaCl}_{2}$ solutions; the resultant curves are shown in Figure 8. It is seen that it was possible to fit the model to represent the experimental data not only for filtrations with deionised water, but also with the other solution environments tested. 
To further trial the model, the $196 \mathrm{kPa}$ filtration data reported by Iritani et al. (2002) were used. The suspensions that Iritani et al. filtered consisted of mixtures of rutile and silica in deionised water at two different $\mathrm{pH}$ values (4.5 and 9.6). At $\mathrm{pH} 4.5$ rutile-silica interactions resulted in a minimum in the specific resistance vs. solids composition trend, and at $\mathrm{pH} 9.6$ the particles were well dispersed. Hence, the model could also be tested on a particle/particle binary mixture for when aggregation does and does not occur, and the resultant fits are shown in Figure 9. As consistent with the rest of this paper, $X_{D}$ is defined as the ratio of volume of the larger solids component (silica in the case of Iritani et al.'s data) to the total volume of solids in the suspension. The experimental points presented in Figure 9 were recalculated from Iritani et al. (2002) because they studied the effects of solids composition in terms of mass fractions. Although a potential drawback of Iritani et al.'s data is that the solids concentration (by volume) is not constant across the solids composition range, this may not be significant for the purpose of testing the model. The model does not contain nor require any explicit information regarding the initial suspension concentration. Instead, as is the case with the Kozeny-Carman equation, information regarding solids concentration is implicitly accounted for via the cake average porosity and solids specific surface.

From Figures 7-9, it is seen that the model represents a wide range of binary suspension filtration data reasonably well using two fitting parameters. The aforementioned data encompass filtrations of fibre/rutile mixtures at various pressures and with different solution environments, as well as filtrations of silica/rutile mixtures at two different $\mathrm{pH}$ values (one induced silica/rutile aggregation and the other did not).

For fibre/rutile binary mixture filtrations, the parameters $b_{1}$ and $b_{2}$ were plotted against filtration pressure (Figure 10). From Figure 10 it is seen that $b_{1}$ values were generally larger than $b_{2}$ values for fibre/rutile mixtures, indicating that the effects (on the filtration performance) of adding rutile to a fibre rich suspension are more significant than adding fibres to a rutile rich suspension. The negative values of $b_{2}$ perhaps indicate that the presence of fibres increases the rutile effective specific surface for rutile rich cakes. This increase in rutile specific surface may be possible via changes in rutile packing brought about by the presence of fibres; as an example, the rutile primary particles could preferentially deposit onto fibre surfaces instead of forming collections of rutile clusters. Alternatively, since the negative values of $b_{2}$ are low in magnitude, it may just be an artefact (experimental and/or fitting) with $b_{2}$ actually being $\sim 0$. With the data of Iritani et al. (2002) for silica/rutile mixtures, with the filtrations at $\mathrm{pH} 4.5$ (aggregation), the values of $b_{1}$ and $b_{2}$ were approximately 2.90 and 0.67 , respectively; and with the filtrations at $\mathrm{pH} 9.6$ (no aggregation), the values of $b_{1}$ and $b_{2}$ were approximately 0.48 and 0.05 , respectively. Here the values of $b_{1}$ and $b_{2}$ are more comparable, particularly when no rutile-silica aggregation was reported ( $\mathrm{pH}$ 9.6). A likely reason for this is the fact that rutile and silica are more comparable in terms of their physical dimensions (size distribution and shape) relative to rutile and fibre. It could be that $b_{1}$ and $b_{2}$ are material specific parameters which are specific to the solids forms in a given solution environment.

It should be noted that the reason for the exponential form in equation (15) was because it fits the data and due to the absence of a more fundamental model. However, considering that equation (16) appears to agree reasonably well with a range of experimental data, the possibility that there may be some underlying fundamental meaning should not be discarded. More rigorous investigation of this possibility, and indeed other approaches of modelling the experimental data which may hold some fundamental basis and/or be of practical use, is beyond the scope of this paper but will be an interesting subject for future work.

\section{CONCLUSIONS}

It was shown that, up to a certain extent, interaction (aggregation) between the two solids components will strongly affect a binary cake's average specific resistance, with the magnitude of 
the effects varying with solids composition. The functional dependence of $\alpha_{a v}$ on $\varepsilon_{a v}$ and the solids specific surface has been shown for binary mixtures, and the effects of aggregation on these parameters highlighted. The ratio of the specific surfaces of the two constituent solids was shown to be a critical parameter in the analysis of aggregating binary suspension filtrations. Difficulties in developing a purely theoretical model to represent the specific resistance trend with solids composition arose due to varying packing mechanisms and aggregation. A semi-empirical model was proposed which represented a wide range of experimental data reasonably well.

The fitting parameters in the semi-empirical model $\left(b_{1}\right.$ and $\left.b_{2}\right)$ may be practically useful for characterisation purposes and/or to increase fundamental understanding of binary suspension filtration. For example, the understanding of how these parameters vary with solids form and the effects of solution environment and filtration pressure on $b_{1}$ and $b_{2}$ could be informative and insightful.

\section{NOTATION}

$b_{1} \quad$ fitting parameter defined by equation (15) (-)

$b_{2} \quad$ fitting parameter defined by equation (15) (-)

$L \quad$ cake thickness $(\mathrm{m})$

$\Delta P \quad$ filtration pressure $(\mathrm{Pa})$

$S_{B} \quad$ ratio of the larger component's specific surface to the smaller component's specific surface in a binary mixture (-)

$S_{m} \quad$ total effective specific surface of solids in a binary mixture $\left(\mathrm{m}^{3} \mathrm{~m}^{-2}\right)$

$S_{i} / \varphi_{i} \quad$ specific surface of the larger $(i=1)$ or smaller $(i=2)$ solids component in contact with the permeating fluid $\left(\mathrm{m}^{3} \mathrm{~m}^{-2}\right)$

$u \quad$ filtrate superficial velocity $\left(\mathrm{m}^{3} \mathrm{~m}^{-1} \mathrm{~s}^{-1}\right)$

$v \quad$ true volume (excluding voids) of filter cake per unit filtration area $\left(\mathrm{m}^{3} \mathrm{~m}^{-2}\right)$

$w \quad$ mass of dry solids deposited per unit area $\left(\mathrm{kg} \mathrm{m}^{-2}\right)$

$X_{D} \quad$ ratio of volume of the larger solids component to the total volume of solids in the binary mixture (-)

$\alpha_{a v} \quad$ average specific resistance of a filter cake $\left(\mathrm{m} \mathrm{kg}^{-1}\right)$

$\mu \quad$ liquid viscosity (Pa s)

$\varepsilon_{a v} \quad$ average filter cake porosity (-)

$\rho_{s} \quad$ solids density $\left(\mathrm{kg} \mathrm{m}^{-3}\right)$

\section{Subscripts}

$s \quad$ referring to solids phase

1 referring to the (larger) solids component 1 (fibres in this work)

2 referring to the (smaller) solids component 2 (rutile in this work)

\section{REFERENCES}

Abe E., Hirosue H. and Yokota K., 1979, Pressure drop through a packed bed of binary Mixture, J. Chem. Eng. Jpn., 12, 302-306.

Abe E. and Hirosue H., 1982, Porosity estimation of a mixed cake in body filtration, J. Chem. Eng. Jpn., 13, 490-493.

Atkins P.W., 1998, Physical Chemistry, $6^{\text {th }}$ Ed., Oxford University Press, Oxford.

Chellappah K., Tarleton E.S. and Wakeman R.J., 2009a, Filtration and sedimentation behaviour of fibre/particle binary suspensions, Filtration, 9(4), 286-294. 
Chellappah K., Tarleton E.S. and Wakeman R.J., 2009b, Constant pressure filtration of fibre/particle mixtures, Proc. Filtech Conference, pp.260-267, 1, Wiesbaden, Germany.

Chellappah K., Tarleton E.S. and Wakeman R.J., 2010, The porosity, permeability and restructuring of heterogeneous filter cakes, Chem. Eng. Technol., 33, 1283-1289.

Donohue T.J. and Wensrich C.M., 2009, Improving permeability prediction for fibrous materials through a numerical investigation into pore size and pore connectivity, Powder Technol., 195, 5762.

Iritani E., Mukai Y. and Toyoda Y., 2002, Properties of a filter cake formed in dead-end microfiltration of binary particulate mixtures, J. Chem. Eng. Jpn., 35, 226-233.

Koenders M.A. and Wakeman R.J., 1997, Filter cake formation from structured suspensions, Chem. Eng. Res. Des., 75, 309-320.

McDonogh R.M., Welsch K., Fane A.G. and Fell C.J.D., 1992, Incorporation of the cake pressure profiles in the calculation of the effect of particle charge on the permeability of filter cakes obtained in the filtration of colloids and particulates, J. Membr. Sci., 72, 197-204.

Shirato M., Sambuichi M. and Okamura S., 1963, Filtration behavior of a mixture of two slurries, AlChE. J., 9, 599-605.

Tarleton E.S. and Hadley R.C., 2003, The application of mechatronic principles in pressure filtration and its impact on filter simulation, Filtration, 3(1), 40-47.

Tarleton E.S., 2008, Cake filter scale-up, simulation and data acquisition - A new approach, J. Chin. Inst. Chem. Eng., 39, 151-160.

Wakeman R.J., Sabri M.N., Tarleton E.S., 1991, Factors affecting the formation and properties of wet compacts, Powder Technol., 65, 283-292.

Wakeman R.J., 1996, Characteristics of filtration using solids added to the suspension as a body aid, Proc. $7^{\text {th }}$ World Filtration Congress, pp.234-238, 1, Budapest, Hungary.

Wakeman R.J. and Akay G., 1997, Membrane-solute and liquid-particle interaction effects in filtration, Filtr. Sep., 34, 511-519.

Wakeman R.J. and Tarleton, E.S., 2005, Solid/Liquid Separation: Principles of Industrial Filtration, Elsevier, Oxford. 


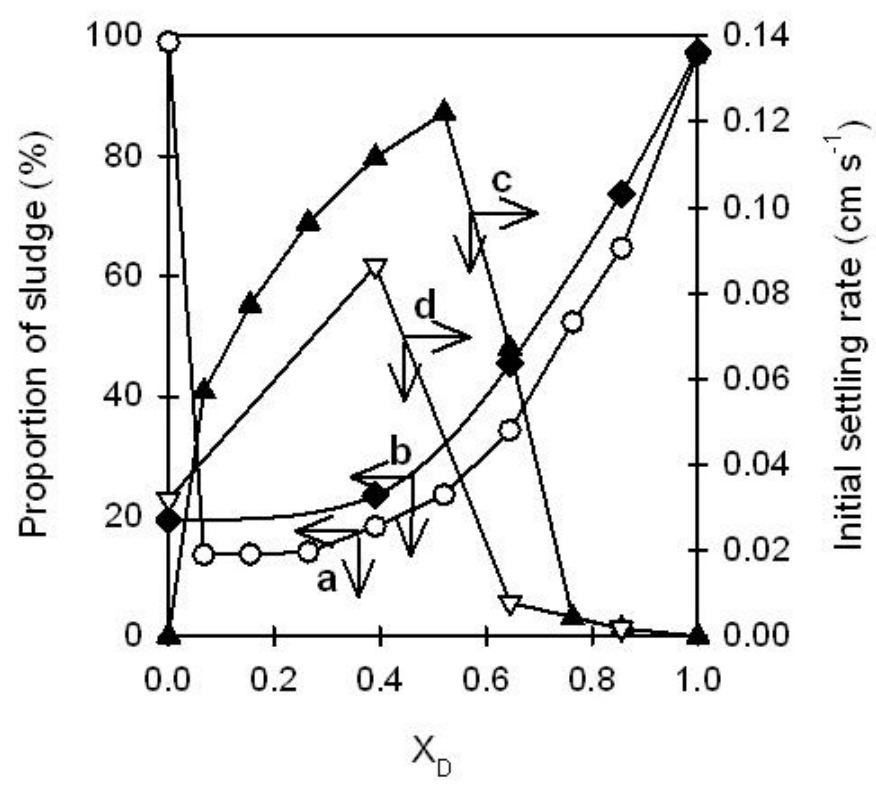

Figure 1: Effects of fibre fraction $\left(X_{D}\right)$ on the proportion of sludge and initial settling rate $(\mathrm{a}=$ proportion of sludge in deionised water; $b=$ proportion of sludge in $0.1 \mathrm{M} \mathrm{CaCl}_{2}$ solution; $c=$ initial settling rate in deionised water; $d=$ initial settling rate in $0.1 \mathrm{M} \mathrm{CaCl}_{2}$ solution).

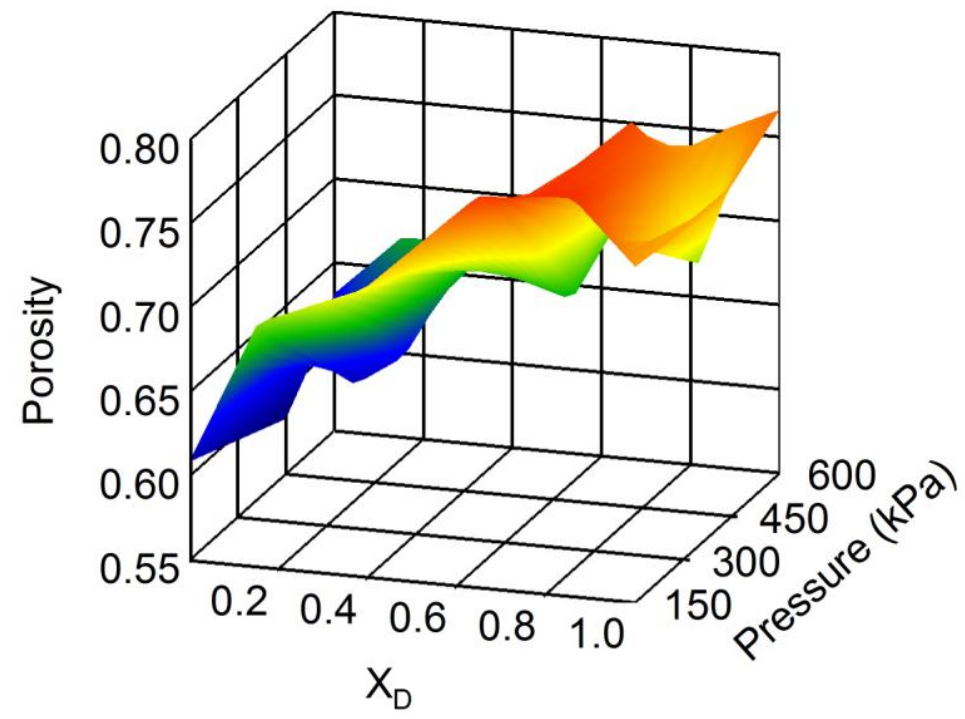

Figure 2: Effects of solids composition and filtration pressure on filter cake average porosity for filtrations from deionised water. 


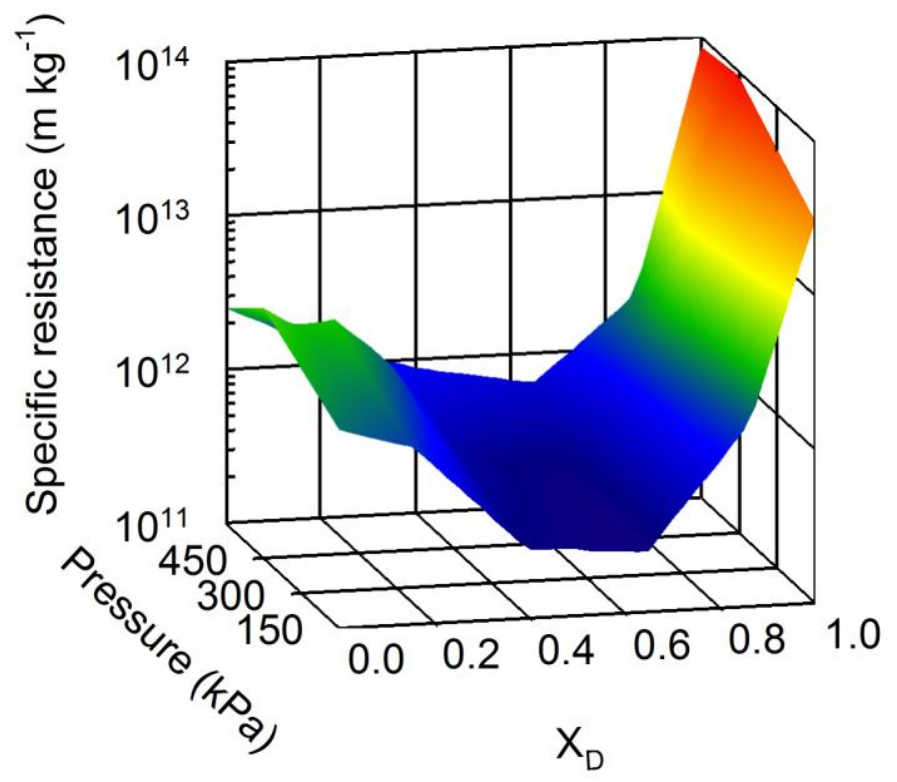

Figure 3: Effects of solids composition and filtration pressure on filter cake average specific resistance for filtrations from deionised water.

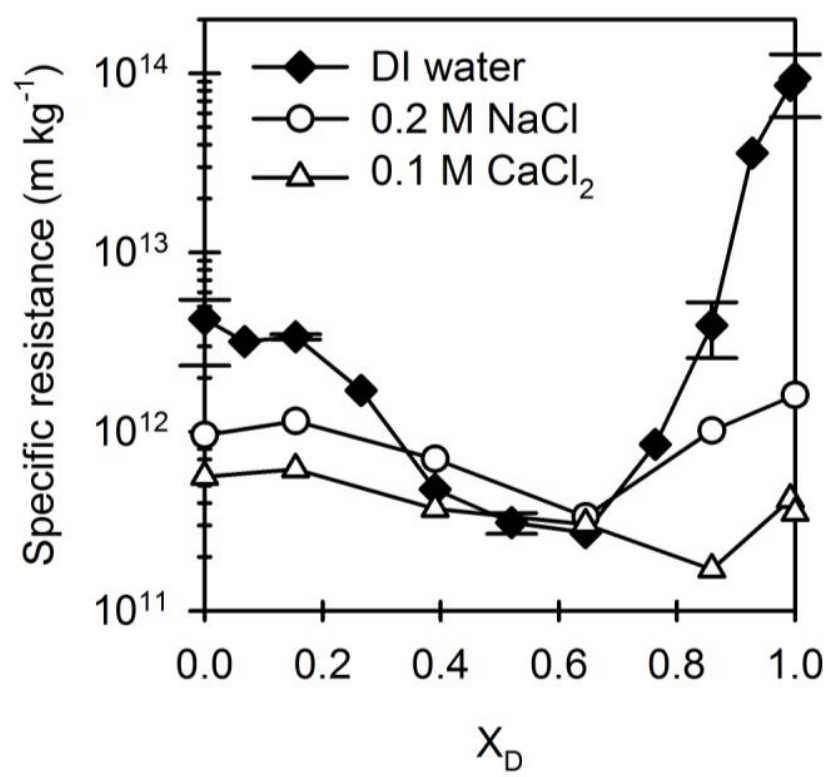

Figure 4: Effects of solids composition and solution environment on filter cake average specific resistance for $450 \mathrm{kPa}$ filtrations. 


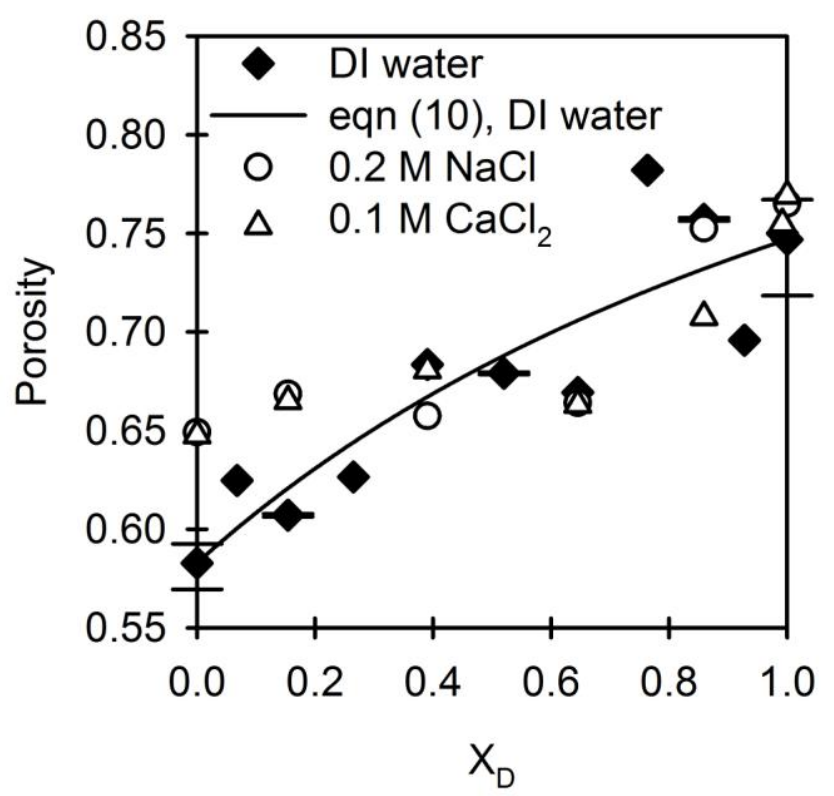

Figure 5: Effects of solids composition and solution environment on filter cake average porosity for $450 \mathrm{kPa}$ filtrations. The prediction given by the additive porosity model (equation (10)) is included for the filtrations from deionised water.

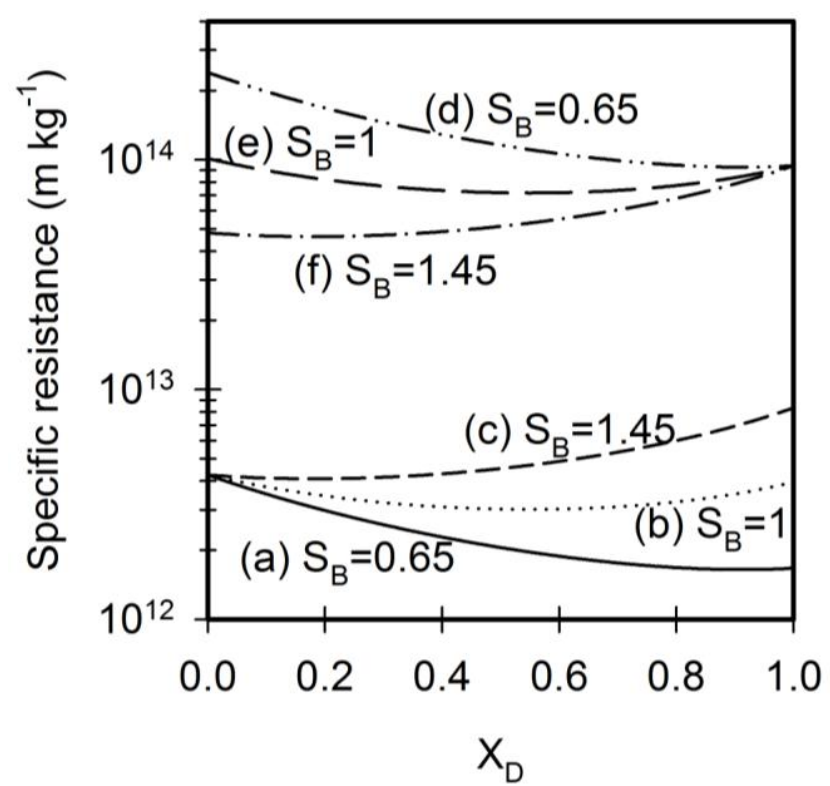

Figure 6: An example family of curves with first $S_{1} / \varphi_{1}$, and then $S_{2} / \varphi_{2}$ altered such that $S_{B}$ falls between 0.65 and 1.45 (the limits set by equation (13) for $450 \mathrm{kPa}$ filtrations from DI water were 0.58 to 1.73$)$. 


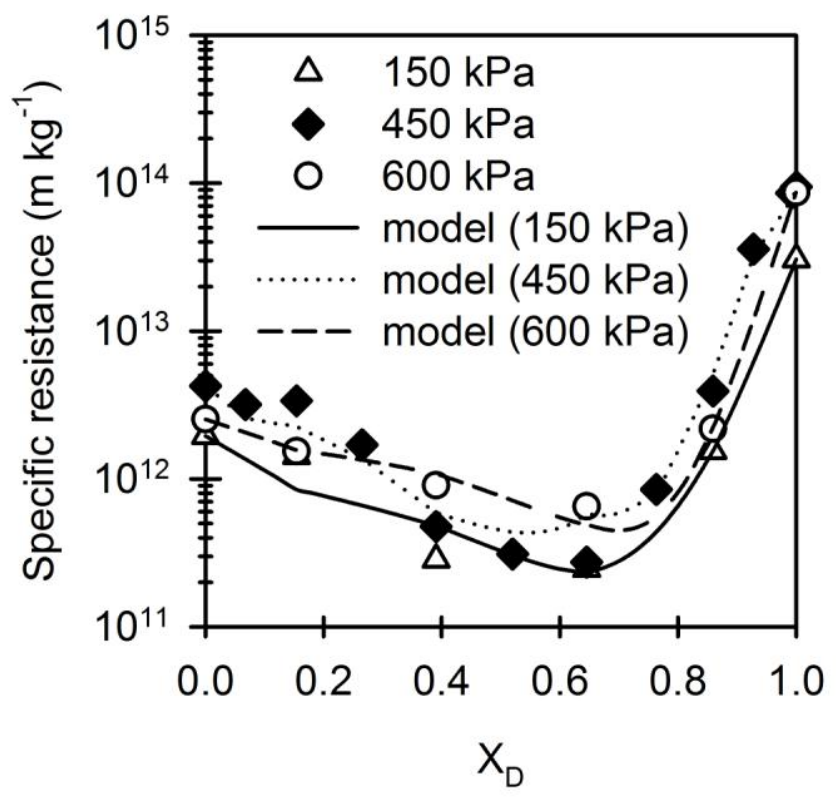

Figure 7: Experimental data for filtrations of fibre/rutile mixtures in deionised water at 150, 450 and $600 \mathrm{kPa}$, and the model fits using equation (16).

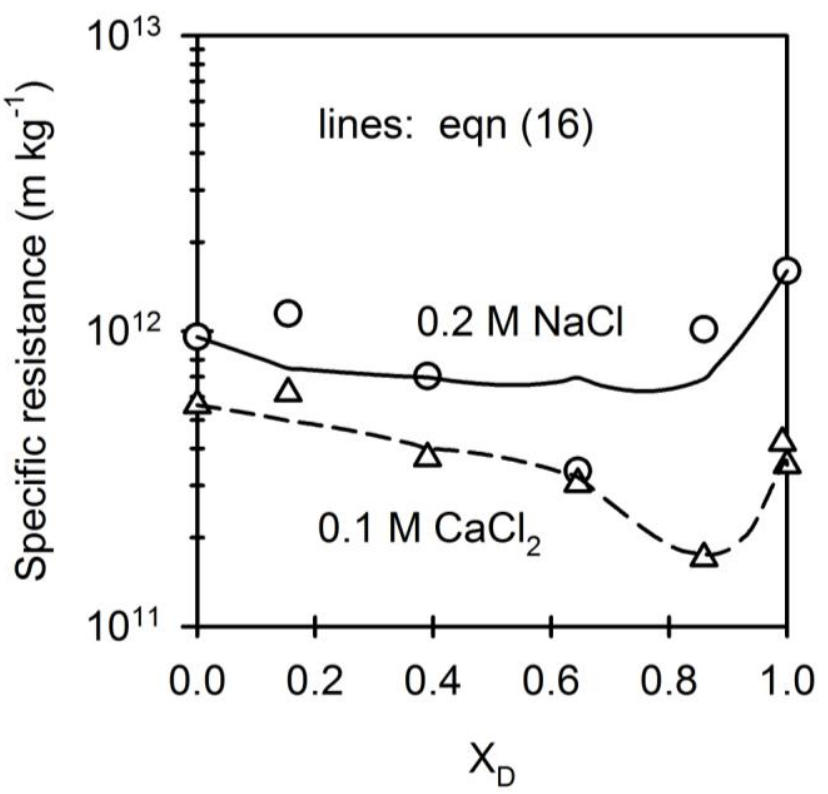

Figure 8: Experimental data for $450 \mathrm{kPa}$ filtrations of fibre/rutile mixtures in $0.2 \mathrm{M} \mathrm{NaCl}$ and $0.1 \mathrm{M}$ $\mathrm{CaCl}_{2}$ solutions, and the model fits using equation (16). 


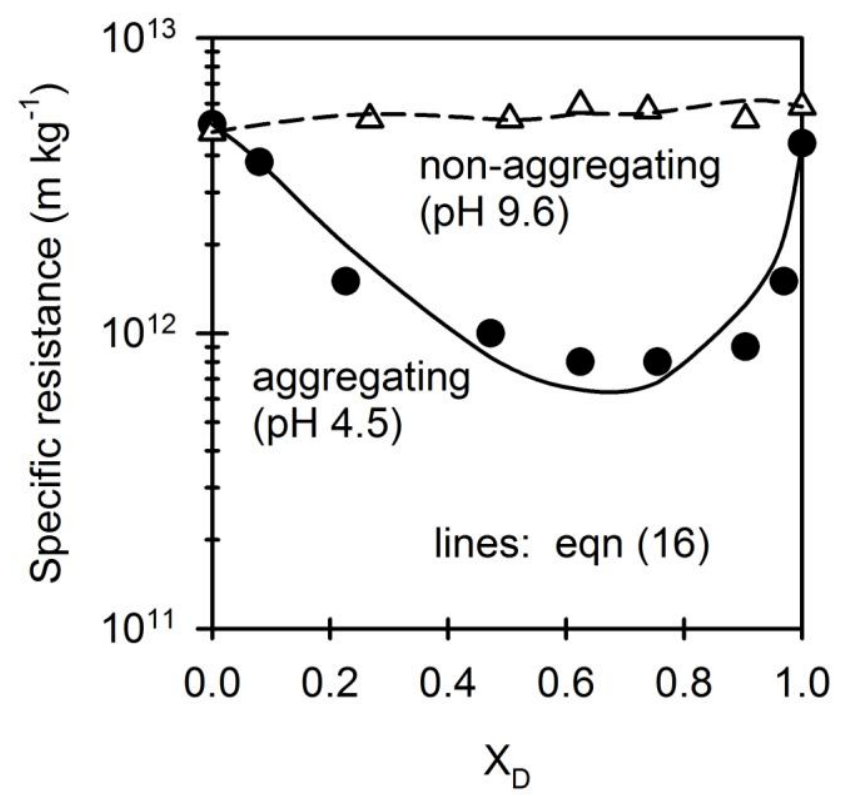

Figure 9: Experimental data for $196 \mathrm{kPa}$ filtrations of silica/rutile mixtures in deionised water at $\mathrm{pH}$ 4.5 (aggregation occurs) and at pH 9.6 (no apparent aggregation), and the model fits using equation (16). In this figure, an $X_{D}$ of 0 refers to pure rutile and an $X_{D}$ of 1 refers to pure silica. The experimental data points were recalculated from those reported by Iritani et al. (2002). The total suspension concentration by volume was not constant here.

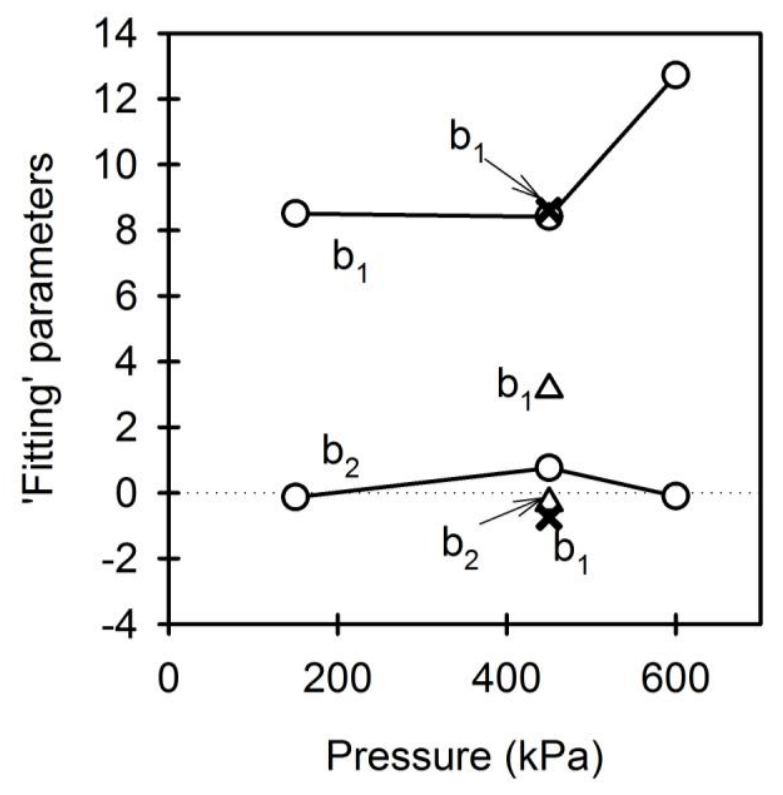

$$
\begin{aligned}
-0 & \text { Dl water } \\
\Delta & 0.2 \mathrm{M} \mathrm{NaCl} \\
\times & 0.1 \mathrm{M} \mathrm{CaCl}_{2}
\end{aligned}
$$

Figure 10: Values of the fitting parameters in equation (16) $\left(b_{1}\right.$ and $\left.b_{2}\right)$ for fibre/rutile binary suspension filtrations with deionised water at three filtration pressures, as well as with $0.2 \mathrm{M} \mathrm{NaCl}$ and $0.1 \mathrm{M} \mathrm{CaCl}_{2}$ solutions at $450 \mathrm{kPa}$. 


\begin{tabular}{|c|c|c|c|c|c|c|c|}
\hline & $\begin{array}{l}10 \% \\
\text { size } \\
(\mu \mathrm{m})\end{array}$ & $\begin{array}{l}50 \% \\
\text { size }^{a} \\
(\mu \mathrm{m})\end{array}$ & $\begin{array}{l}90 \% \\
\text { size } \\
(\mu \mathrm{m})\end{array}$ & Aspect ratio & $\begin{array}{c}\text { 万-potential } \\
\text { in DI water } \\
(\mathrm{mV})\end{array}$ & $\begin{array}{c}\zeta \text {-potential } \\
\text { in } 0.2 \mathrm{M} \\
\mathrm{NaCl}(\mathrm{mV})\end{array}$ & $\begin{array}{c}\zeta \text {-potential in } \\
0.1 \mathrm{M} \mathrm{CaCl}_{2} \\
(\mathrm{mV})\end{array}$ \\
\hline Rutile & 0.4 & 0.45 & 0.7 & $2-4$ & -35 & -30 & +21 \\
\hline Fibres & 6 & 15 & 29 & $\sim 100$ & -18 & -20 & -5 \\
\hline
\end{tabular}

${ }^{a}$ width for fibres

Table 1: Relevant properties of the two solids used. 